Pacific

Journal of

Mathematics

PARTIAL INTEGRABILITY OF ALMOST COMPLEX STRUCTURES AND THE EXISTENCE OF SOLUTIONS FOR QUASILINEAR CAUCHY-RIEMANN EQUATIONS

ChONG-Kyu HAN AND Jong-Do PARK

Volume $265 \quad$ No. 1

September 2013 


\title{
PARTIAL INTEGRABILITY OF ALMOST COMPLEX STRUCTURES AND THE EXISTENCE OF SOLUTIONS FOR QUASILINEAR CAUCHY-RIEMANN EQUATIONS
}

\author{
CHONG-KYU HAN AND JONG-Do PARK
}

\begin{abstract}
We study the local solvability of the system of quasilinear Cauchy-Riemann equations for $\boldsymbol{d}$ unknown functions in $\boldsymbol{n}$ complex variables, which is a system of elliptic type and overdetermined if $n \geq 2$. We consider an associated almost complex structure on $\mathbb{C}^{n+d}$ and its partial integrability and prove by using the Newlander-Nirenberg theorem and its algebraic generalizations that the existence of a pseudoholomorphic function on the zero set is equivalent to the local solvability of the original quasilinear system. We discuss an algorithm for finding pseudoholomorphic functions on the zero set and then present examples.
\end{abstract}

\section{Introduction}

A classical method for solving partial differential equations (PDE) of first order is the method of characteristics which originated from [Monge 1803]. One finds curves along which a PDE becomes a system of ordinary differential equations and constructs a solution whose graph, or 1-jet graph, is a union of those curves. Consider a quasilinear equation

$$
\sum_{\lambda=1}^{n} a^{\lambda}(x, u) \frac{\partial u}{\partial x^{\lambda}}=b(x, u)
$$

for a real-valued function $u$ in $n$ real variables $x=\left(x^{1}, \ldots, x^{n}\right)$, where $a^{\lambda}$ and $b$ are smooth $\left(C^{\infty}\right)$ and $a^{\lambda}, \lambda=1, \ldots, n$, are not all zero. The characteristic vector field of $(0-1)$ is a smooth vector field

$$
X=\sum_{\lambda=1}^{n} a^{\lambda} \frac{\partial}{\partial x^{\lambda}}+b \frac{\partial}{\partial u}
$$

The authors were partially supported by National Research Foundation (NRF) of Republic of Korea, with grant numbers 2009-0070971 and 2010-0011841, respectively.

MSC2010: primary 32W05, 35N10; secondary 32Q60, 35J60.

Keywords: overdetermined system, elliptic PDE system, almost complex structure, nonlinear

Cauchy-Riemann equations. 
on $\mathbb{R}^{n+1}=\{(x, u)\}$ and a smooth real-valued function $\phi(x, u)$ is a first integral of $X$ if $X \phi=0$. Then, by the implicit function theorem, any first integral $\phi$ with $\phi_{u} \neq 0$ gives an implicit solution $\phi(x, u)=0$ to $(0-1)$. The same method works for systems. Consider

$$
\sum_{\lambda=1}^{n} a_{j}^{\lambda}(x, u) \frac{\partial u}{\partial x^{\lambda}}=b_{j}(x, u), \quad j=1, \ldots, p, \quad p \leq n,
$$

for a real-valued function $u$ in $n$ real variables $x=\left(x^{1}, \ldots, x^{n}\right)$. We assume the matrix $\left(a_{j}^{\lambda}\right)$ has maximal rank $p$. If $p \geq 2$, then (0-2) is overdetermined; therefore, there are no solutions generically. To discuss the existence of solutions let

$$
X_{j}=\sum_{\lambda=1}^{n} a_{j}^{\lambda} \frac{\partial}{\partial x^{\lambda}}+b_{j} \frac{\partial}{\partial u}, \quad j=1, \ldots, p,
$$

be vector fields on $\mathbb{R}^{n+1}=\{(x, u)\}$. For a smooth function $u(x)$, a normal vector to the graph $S=\left\{(x, u(x)) \in \mathbb{R}^{n+1}\right\}$ is $(\nabla u,-1)=\left(\partial u / \partial x^{1}, \ldots, \partial u / \partial x^{n},-1\right)$. Then (0-2) is equivalent to $X_{j} \cdot(\nabla u,-1)=0$, which implies that $X_{j}$ is tangent to the graph $S$ at every point. A smooth real-valued function $F$ is said to have invariant zero-level with respect to vector fields $X_{1}, \ldots, X_{p}$ if $\left(X_{j} F\right)(x)=0$ for all $j=1, \ldots, p$ and for all $x$ with $F(x)=0$. We have:

Theorem 0.1. Let $\left(x_{0}, u_{0}\right) \in \mathbb{R}^{n} \times \mathbb{R}$. On a neighborhood of $\left(x_{0}, u_{0}\right)$ there exists a solution $u(x)$ of (0-2) with $u\left(x_{0}\right)=u_{0}$ if and only if there is a function $F(x, u)$ with $\partial F / \partial u \neq 0$ and $F\left(x_{0}, u_{0}\right)=0$ that has invariant zero-level with respect to the set of vector fields (0-3).

Proof. Suppose that $u=f(x), x=\left(x^{1}, \ldots, x^{n}\right)$, is a solution of (0-2). Let $F(x, u):=f(x)-u$. Then we see that $F_{u} \neq 0$ and that, for each $j=1, \ldots, p$,

$$
X_{j} F=0 \quad \text { on }\{F=0\} .
$$

Conversely, suppose that $F(x, u)$ is a function with $F_{u} \neq 0, F\left(x_{0}, u_{0}\right)=0$, that has invariant zero-level. Differentiating the implicit function $F(x, u)=0$ with respect to $x^{\lambda}$ using the chain rule, we have

$$
\frac{\partial F}{\partial x^{\lambda}}=-\frac{\partial F}{\partial u} \frac{\partial u}{\partial x^{\lambda}} \quad \text { for each } \lambda=1, \ldots, n .
$$

On the other hand, since $F$ has invariant zero-level we have

$$
X_{j} F=\sum_{\lambda=1}^{n} a_{j}^{\lambda} \frac{\partial F}{\partial x^{\lambda}}+b_{j} \frac{\partial F}{\partial u}=0 \quad \text { on }\{F=0\} .
$$


Substituting (0-4) for $\partial F / \partial x^{\lambda}$ in (0-5) we have

$$
-\frac{\partial F}{\partial u}\left(\sum_{\lambda=1}^{n} a_{j}^{\lambda} \frac{\partial u}{\partial x^{\lambda}}-b_{j}\right)=0 \quad \text { on }\{F=0\}
$$

that is, $u=f(x)$ satisfies $(0-2)$.

For more details on (0-2) we refer the readers to [Han and Park 2013]. In this paper we study the complex analogue of Theorem 0.1 . Let $z=\left(z^{1}, \ldots, z^{n}\right)$ for $z^{j}=x^{j}+\sqrt{-1} y^{j}$ be complex variables and let

$$
\frac{\partial}{\partial \bar{z}^{j}}:=\frac{1}{2}\left(\frac{\partial}{\partial x^{j}}+\sqrt{-1} \frac{\partial}{\partial y^{j}}\right) \text { and } \frac{\partial}{\partial z^{j}}:=\frac{1}{2}\left(\frac{\partial}{\partial x^{j}}-\sqrt{-1} \frac{\partial}{\partial y^{j}}\right) .
$$

Consider a system of PDE for a complex-valued unknown function $w=w(z, \bar{z})$ :

$$
\frac{\partial w}{\partial \bar{z}^{j}}+\sum_{k=1}^{n} A_{j}^{k}(z, \bar{z}, w, \bar{w}) \frac{\partial w}{\partial z^{k}}=B_{j}(z, \bar{z}, w, \bar{w}), \quad j=1, \ldots, n,
$$

where $A_{j}^{k}$ and $B_{j}$ are complex-valued $C^{\infty}$ functions that are defined on a neighborhood of the origin of $\mathbb{C}^{n+1}=\{(z, w)\}$ and $A_{j}^{k}$ are sufficiently small. If $n \geq 2$, then (0-6) is overdetermined. We shall call (0-6) quasilinear Cauchy-Riemann equations. We prove the local solvability in the $C^{\infty}$ category by purely formal arguments based on the Newlander-Nirenberg theorem and its algebraic generalizations. We observed that a function $\zeta(z, \bar{z}, w, \bar{w})=0$ is an implicit solution to (0-6) if and only if $\zeta$ is pseudoholomorphic on the zero set (see Definition 1.6) with respect to an almost complex structure $J$ on $\mathbb{C}^{n+1}=\{(z, w)\}$ determined by the coefficients $A_{j}^{k}$ and $B_{j}$ (Theorem 3.3). Another observation is that a function $\zeta(z, \bar{z}, w, \bar{w})$ with $d \zeta \wedge d \bar{\zeta} \neq 0$ is pseudoholomorphic on the zero set if and only if the zero locus $\zeta=0$ is a $J$-invariant submanifold of $\left(\mathbb{C}^{n+1}, J\right)$ (Theorem 2.5). To check the partial integrability of the almost complex structure we make use of Theorem 1.3, which is due to L. Nirenberg and F. Treves.

Section 4 is a generalization of our results of Section 3 to the cases of multiple unknown functions $w=\left(w^{1}, \ldots, w^{d}\right)$ :

$$
\frac{\partial w^{\alpha}}{\partial \bar{z}^{j}}+\sum_{k=1}^{n} A_{j}^{k}(z, \bar{z}, w, \bar{w}) \frac{\partial w^{\alpha}}{\partial z^{k}}=B_{j}^{\alpha}(z, \bar{z}, w, \bar{w})
$$

for each $j=1, \ldots, n(n \geq 1)$ and $\alpha=1, \ldots, d$, where $A_{j}^{k}$ and $B_{j}^{\alpha}$ are $C^{\infty}$ functions defined on a neighborhood of the origin of $\mathbb{C}^{n+d}$ and $A_{j}^{k}$ are sufficiently small.

We discuss in Section 5 the determined case $n=1$. In Section 6 we present examples of $n=2$ including the equations for the pseudoanalytic functions, which will be introduced in Section 7. In the last section of this paper we briefly survey 
the history of the perturbed Cauchy-Riemann equations and overdetermined PDE systems.

Finally we mention the regularity of solutions to (0-6) or (0-7): it is well-known (see [Gilbarg and Trudinger 1998]) that a linear elliptic partial differential equation is hypoelliptic; that is, any distribution solution is $C^{\infty}$ whenever all the coefficients of the differential operators are $C^{\infty}$. In general, a nonlinear differential equation is said to be elliptic if its linearization is an elliptic differential operator (see [Taylor 1997]). It was proved in [Douglis and Nirenberg 1955] that nonlinear elliptic systems are hypoelliptic by Schauder-type a priori estimates. Since the linearization of each quasilinear equation in (0-6) or (0-7) is elliptic, any $C^{1, \alpha}$ solution to (0-6) or (0-7) is always $C^{\infty}$.

\section{1. $J$-holomorphic functions on almost complex manifolds}

Let $M$ be a smooth $\left(C^{\infty}\right)$ manifold of dimension $2 m, m \geq 2$, with smooth almost complex structure $J$. Then the complexified tangent bundle $\mathbb{C} T M$ has the decomposition

$$
\mathbb{C} T M=T^{1,0}(M) \oplus T^{0,1}(M),
$$

where $T^{1,0}(M)\left(T^{0,1}(M)\right.$, respectively) is the subbundle of rank- $m$ of eigenvectors of $J$ associated with the eigenvalue $i$ ( $-i$, respectively). The dual decomposition of the complexified cotangent bundle $\mathbb{C} T^{*} M$ is

$$
\mathbb{C} T^{*} M=\left(T^{*} M\right)^{1,0} \oplus\left(T^{*} M\right)^{0,1} .
$$

We can find real vector fields $X_{j}, j=1, \ldots, m$, such that

$$
X_{1}, J X_{1}, \ldots, X_{m}, J X_{m}
$$

spans the real tangent bundle $T M$. Let

$$
Z_{j}=\frac{1}{2}\left(X_{j}-i J X_{j}\right) \quad \text { and } \quad \bar{Z}_{j}=\frac{1}{2}\left(X_{j}+i J X_{j}\right)
$$

for each $j=1, \ldots, m$. Then $\left\{Z_{1}, \ldots, Z_{m}\right\}$ spans $T^{1,0}(M)$ and $\left\{\bar{Z}_{1}, \ldots, \bar{Z}_{m}\right\}$ spans $T^{0,1}(M)$. Let $\left\{\theta^{1}, \ldots, \theta^{m}\right\}$ be a set of independent 1 -forms that annihilates $T^{0,1}(M)$ and thus $\left\{\bar{\theta}^{1}, \ldots, \bar{\theta}^{m}\right\}$ annihilates $T^{1,0}(M)$. Then the subbundles $\left(T^{*} M\right)^{1,0}$ and $\left(T^{*} M\right)^{0,1}$ of the complexified cotangent bundle are the linear spans of $\left\{\theta^{1}, \ldots, \theta^{m}\right\}$ and $\left\{\bar{\theta}^{1}, \ldots, \bar{\theta}^{m}\right\}$, respectively.

A complex-valued function $\zeta$ is called $J$-holomorphic (or pseudoholomorphic) if

$$
\bar{Z}_{j} \zeta=0, \quad j=1, \ldots, m .
$$

Equation (1-1) is an overdetermined system of linear PDE, and thus, in general, there are no solutions other than constants. $J$-holomorphic functions $\zeta^{1}, \ldots, \zeta^{q}$ 
are said to be independent if

$$
d \zeta^{1} \wedge \cdots \wedge d \zeta^{q} \neq 0
$$

Equation (1-1) is equivalent to saying that $d \zeta$ is a section of $\left(T^{*} M\right)^{1,0}$, so that there exist at most $m$ independent $J$-holomorphic functions. $J$ is said to be integrable if

$$
\left[T^{1,0}(M), T^{1,0}(M)\right] \subset T^{1,0}(M),
$$

which means that the bracket of any two sections of $T^{1,0}(M)$ is again a section of $T^{1,0}(M)$. For the theory of general integrable structures, we refer the readers to [Berhanu et al. 2008].

We consider the exterior algebra of differential forms with complex coefficients:

$$
\Omega^{*}=\Omega^{0} \oplus \Omega^{1} \oplus \cdots \oplus \Omega^{2 m},
$$

where $\Omega^{0}$ is the ring of smooth complex-valued functions and $\Omega^{r}(r=1, \ldots, 2 m)$ is the module over $\Omega^{0}$ of complex-valued smooth $r$-forms on $M$.

Definition 1.1. A subalgebra $\mathscr{I}$ of $\Omega^{*}$ is called an algebraic ideal if the following conditions hold:

(i) $\mathscr{I} \wedge \Omega^{*} \subset \mathscr{I}$,

(ii) if $\phi=\sum_{r=0}^{2 m} \phi_{r} \in \mathscr{I}$, where $\phi_{r} \in \Omega^{r}$, then each component $\phi_{r}$ belongs to $\mathscr{I}$ (homogeneity condition).

The homogeneity condition implies that $\mathscr{I}$ is a two-sided ideal; that is, $\Omega^{*} \wedge \mathscr{I} \subset \mathscr{I}$.

In this paper we consider ideals generated by finitely many complex-valued functions and finitely many 1 -forms. Let $\rho=\left(\rho^{1}, \ldots, \rho^{d}\right)$ and $\phi=\left(\phi^{1}, \ldots, \phi^{q}\right)$ be a system of functions and 1-forms, respectively. We denote by $\mathscr{I}(\rho, \phi)$, or simply by $(\rho, \phi)$, the algebraic ideal generated by $\rho$ and $\phi$, which is the set of all elements of $\Omega^{*}$ of the form

$$
\sum_{\alpha=1}^{d} \rho^{\alpha} \omega^{\alpha}+\sum_{k=1}^{q} \phi^{k} \wedge \psi^{k} \quad \text { for some } \omega^{\alpha}, \psi^{k} \in \Omega^{*} .
$$

For two elements $\alpha$ and $\beta$ of $\Omega^{*}$,

$$
\alpha \equiv \beta \bmod (\rho, \phi)
$$

means that $\alpha-\beta \in \mathscr{I}(\rho, \phi)$.

The integrability condition (1-2) can be written as

$$
\left[Z_{j}, Z_{k}\right] \in \Gamma\left(T^{1,0}(M)\right) \text { for all } j, k=1, \ldots, m,
$$

where $\Gamma$ denotes the set of all smooth sections. Equation (1-3) is equivalent to

$$
d \theta^{\ell} \equiv 0 \bmod (\theta) \quad \text { for all } \ell=1, \ldots, m,
$$


where $\theta=\left(\theta^{1}, \ldots, \theta^{m}\right)$.

Theorem 1.2 [Newlander and Nirenberg 1957]. Let $\left(M^{2 m}, J\right)$ be a $C^{\infty}$ almost complex manifold. If $J$ is integrable then there exist $m$ independent $J$-holomorphic functions.

The converse is also true, which is rather trivial. Now we fix notations: for any subbundle $I \subset\left(T^{*} M\right)^{1,0}$ we denote by $\underline{I}$ the module over $\Omega^{0}$ of smooth sections of $I$ and by $(I)$ the algebraic ideal of $\Omega^{*}$ generated by the smooth sections of $I$. By using Theorem 1.2 and the Frobenius theorem the following was proved in [Trèves 1981]:

Theorem 1.3. Suppose that $T^{\prime}$ is a subbundle of $\left(T^{*} M\right)^{1,0}$ of rank $q(q<m)$ and that $T^{\prime}$ is closed; that is, $d \underline{T}^{\prime} \subset\left(T^{\prime}\right)$. Then there exist $q$ independent $J$-holomorphic functions $\zeta^{1}, \ldots, \zeta^{q}$ whose differentials $d \zeta^{1}, \ldots, d \zeta^{q}$ span $T^{\prime}$.

The problem of determining conditions for the existence of $J$-holomorphic functions on almost complex manifolds has been examined in [Mushkarov 1981; 1986] by studying the involutivity of the Nijenhuis bundle. Criteria for the existence of $J$-holomorphic mappings into other almost complex manifolds are given in [Kruglikov 1998] in terms of Nijenhuis tensors and their generalizations. The following theorem is found in [Han and Kim 2012].

Theorem 1.4. Let $M^{2 m}(m \geq 2)$ be a $C^{\infty}$ manifold with $C^{\infty}$ almost complex structure $J$. Let $\left(T^{*} M\right)^{1,0}$ be the bundle of $(1,0)$-forms. Then there exist a sequence of subbundles $\left(T^{*} M\right)^{1,0}:=I^{(0)} \supset I^{(1)} \supset I^{(2)} \supset \cdots$ and a nonnegative integer $v$ such that for $k=0,1,2, \ldots$ :

(i) $I^{(k+1)} \subsetneq I^{(k)}$, if $k<v$,

(ii) $I^{(k+1)}=I^{(k)}$, if $k \geq v$,

(iii) $d \underline{I}^{(k+1)} \equiv 0 \bmod I^{(k)}$,

under a generic assumption in each step of the construction of the sequence. Moreover, a function $f$ is $J$-holomorphic if and only if $d f \in I^{(v)}$; thus the number of independent $J$-holomorphic functions is equal to the rank of $I^{(v)}$.

Definition 1.5. The integer $v$ of Theorem 1.4 is called the type of the almost complex structure $J$. We also say that the Pfaffian system $\left(\theta^{1}, \ldots, \theta^{m}\right)$ has derived length $v$.

Proof of Theorem 1.4. We shall find the largest closed subbundle of $\left(T^{*} M\right)^{1,0}$ starting with $I=I^{(0)}=\left(T^{*} M\right)^{1,0}$ : the exterior derivative $d: \underline{I} \rightarrow \Omega^{2}$ is not a module homomorphism, but composition with the projection

$$
\stackrel{d}{\rightarrow} \Omega^{2} \stackrel{\pi}{\rightarrow} \Omega^{2} /(I)
$$


is an $\Omega^{0}$-module homomorphism. Let $\delta=\pi \circ d$. Consider the submodule $\underline{I}^{(1)}:=$ $\operatorname{ker} \delta$ of $\underline{I}$. We assume that $\underline{I}^{(1)}$ has constant rank on $M$, and hence defines a subbundle $I^{(1)}$ of $\left(T^{*} M\right)^{1,0}$. We have a short exact sequence of $\Omega^{0}$-modules

$$
0 \rightarrow \underline{I}^{(1)} \rightarrow \underline{I} \stackrel{\delta}{\rightarrow} d \underline{I} /(I) \rightarrow 0 .
$$

The subbundle $I^{(1)}$ is called the first derived system of $\left(T^{*} M\right)^{1,0}$. Assuming that $\underline{I}^{(k-1)}$ has constant rank, we define inductively the $k$-th derived system $I^{(k)}$ by

$$
0 \rightarrow \underline{I}^{(k)} \rightarrow \underline{I}^{(k-1)} \stackrel{\delta}{\rightarrow} d \underline{I}^{(k-1)} /\left(I^{(k-1)}\right) \rightarrow 0 .
$$

Let $v$ be the smallest integer with $I^{(v)}=I^{(v+1)}$. Then we have the sequence of subbundles

$$
\left(T^{*} M\right)^{1,0}:=I:=I^{(0)} \supset I^{(1)} \supset \cdots \supset I^{(\nu-1)} \supset I^{(v)} .
$$

Notice that $d \underline{I}^{(v)} \subset\left(I^{(v)}\right)$; that is, $I^{(v)}$ is closed. Assume that $\underline{I}^{(v)}$ has constant rank $q$. Then by Theorem 1.3 there exist independent $J$-holomorphic functions $\zeta^{1}, \ldots, \zeta^{q}$, which completes the proof of Theorem 1.4.

The idea of Theorem 1.4 came from the theory of first integrals for Pfaffian systems due to E. Cartan and R. Gardner [Gardner 1967], which is a real version of Theorem 1.4. A generalized notion of the first integral has been used in [Ahn and Han 2012; Han and Park 2013]. Our standard reference for the theory of Pfaffian systems is [Bryant et al. 1991]. In this paper we need a notion of $J$-holomorphicity on the zero set that we define as follows:

Definition 1.6. A system of complex-valued functions $\zeta=\left(\zeta^{1}, \ldots, \zeta^{d}\right)$ is said to be $J$-holomorphic on the zero set if, for each $\alpha=1, \ldots, d$, we have $\left(\bar{Z}_{j} \zeta^{\alpha}\right)(x)=0$, $j=1, \ldots, m$, for all $x$ with $\zeta(x)=0$, or, equivalently, if

$$
d \zeta^{\alpha} \equiv 0 \bmod (\zeta, \bar{\zeta}, \theta)
$$

Assuming further that $\theta^{j}$ are dual to $Z_{k}$, that is,

$$
\theta^{j}\left(Z_{k}\right)=\delta_{k}^{j},
$$

we define $\partial f$ and $\bar{\partial} f$ for any complex-valued function $f$ by

$$
\partial f:=\sum_{j=1}^{m}\left(Z_{j} f\right) \theta^{j}, \quad \bar{\partial} f:=\sum_{j=1}^{m}\left(\bar{Z}_{j} f\right) \bar{\theta}^{j} .
$$

Then we have

$$
d f=\partial f+\bar{\partial} f .
$$


We may write (1-5) as

$$
\bar{\partial} \zeta^{\alpha} \equiv 0 \bmod (\zeta, \bar{\zeta}) \quad \text { for each } \alpha=1, \ldots, d
$$

\section{2. $J$-invariant submanifolds}

A submanifold $N \subset M$ is said to be $J$-invariant if $J T_{x} N=T_{x} N$ at every point $x \in N$. $J$-invariant submanifolds are even dimensional. In this section we shall discuss the properties of a system of real-valued functions

$$
\rho=\left(\rho^{1}, \ldots, \rho^{2 d}\right)
$$

that defines a $J$-invariant submanifold $N$. The system $\rho$ shall be called nondegenerate if

$$
d \rho^{1} \wedge \cdots \wedge d \rho^{2 d} \neq 0
$$

Given a set of finitely many differential 1 -forms $\left\{\phi^{1}, \phi^{2}, \ldots,\right\}$, we shall mean by the rank at $x \in M$ the number of independent 1 -forms at $x$.

Now we consider a submanifold $N^{2 n}$ of $\left(M^{2 m}, J\right)$ locally defined as the common zero set of a nondegenerate set of real-valued functions $\rho^{1}, \ldots, \rho^{2 d}$ with $d=m-n$. Proposition 2.1. Suppose that $\left(\rho^{1}, \ldots, \rho^{2 d}\right)$ is a nondegenerate set of real-valued functions on a neighborhood of a point $x$ of $\left(M^{2 m}, J\right)$ with $d \leq m$. Then we have

$$
d \leq \operatorname{rank}\left(\partial \rho^{1}, \ldots, \partial \rho^{2 d}\right) \leq 2 d .
$$

Proof. Consider

$$
\begin{aligned}
d \rho^{1} \wedge \cdots \wedge d \rho^{2 d} & =\left(\partial \rho^{1}+\bar{\partial} \rho^{1}\right) \wedge \cdots \wedge\left(\partial \rho^{2 d}+\bar{\partial} \rho^{2 d}\right) \\
& =\left(\partial \rho^{1} \wedge \cdots \wedge \partial \rho^{2 d}\right)+\text { mixed terms }+\left(\bar{\partial} \rho^{1} \wedge \cdots \wedge \bar{\partial} \rho^{2 d}\right)
\end{aligned}
$$

where "mixed terms" means those terms that contain both $\partial \rho^{\alpha}$ 's and $\bar{\partial} \rho^{\alpha}$ 's. If $\operatorname{rank}\left(\partial \rho^{1}, \ldots, \partial \rho^{2 d}\right) \leq d-1$ then each term in the last line of (2-1) contains either $\partial \rho^{\alpha}$ 's more than $d$ times or $\bar{\partial} \rho^{\alpha}$ 's more than $d$ times. Hence, each term of the last line of (2-1) is zero at $x$, which contradicts the nondegeneracy condition.

Proposition 2.2. Let u be a $C^{\infty}$ complex-valued function on $M$ and $X \in T M$. Then $\partial u(X)=\frac{1}{2}\{d u(X)-\sqrt{-1} d u(J X)\} \quad$ and $\quad \bar{\partial} u(X)=\frac{1}{2}\{d u(X)+\sqrt{-1} d u(J X)\}$.

Proof. Since $\partial u$ annihilates any $(0,1)$-vector, we have

$$
\begin{aligned}
\partial u(X) & =\partial u\left(X^{1,0}+X^{0,1}\right)=\partial u\left(X^{1,0}\right)=d u\left(X^{1,0}\right) \\
& =\frac{1}{2} d u\{X-\sqrt{-1} J X\}=\frac{1}{2}\{d u(X)-\sqrt{-1} d u(J X)\} .
\end{aligned}
$$

We prove the second equality similarly. 
Theorem 2.3. Let $N^{2 n}$ be a submanifold of $\left(M^{2 m}, J\right)$ given as a common zero set of a nondegenerate system of real-valued functions $\rho^{1}, \ldots, \rho^{2 d}$ with $d=m-n$. Let $T^{1,0} N=\{X-\sqrt{-1} J X: X \in T N \cap J T N\}$ and $T^{0,1} N=\{X+\sqrt{-1} J X$ : $X \in T N \cap J T N\}$. Then the following are equivalent:

(i) $\mathrm{N}$ is $\mathrm{J}$-invariant.

(ii) $T_{x}^{1,0} N$ and $T_{x}^{0,1} N$ have complex dimension $n$ for each $x \in N$.

(iii) $\operatorname{rank}\left(\partial \rho^{1}, \ldots, \partial \rho^{2 d}\right)(x)=d$ for each $x \in N$.

Proof. (i) $\Rightarrow$ (ii): Suppose that $N$ is $J$-invariant. Then it is easy to see that there exist linearly independent real vector fields

$$
X_{1}, J X_{1}, \ldots, X_{n}, J X_{n}
$$

that are tangent to $N$. Thus $2 n$ complex vectors $X_{k}^{1,0}:=\frac{1}{2}\left(X_{k}-\sqrt{-1} J X_{k}\right)$ and $X_{k}^{0,1}:=\frac{1}{2}\left(X_{k}+\sqrt{-1} J X_{k}\right), k=1, \ldots, n$, are linearly independent and tangent to $N$, which implies (ii).

(ii) $\Rightarrow$ (iii): Suppose that, for each $x \in N, T_{x}^{1,0} N$ has complex dimension $n$. Since

$$
\begin{aligned}
T^{1,0} N & =\left\{Z \in T^{1,0} M: d \rho^{\alpha}(Z)=\partial \rho^{\alpha}(Z)=0, \alpha=1, \ldots, 2 d\right\} \\
& =\bigcap_{\alpha=1}^{2 d}\left(\operatorname{Ker} \partial \rho^{\alpha} \cap T^{1,0} M\right)=\left(\bigcap_{\alpha=1}^{2 d}\left(\operatorname{Ker} \partial \rho^{\alpha}\right)\right) \cap T^{1,0} M
\end{aligned}
$$

has a fiber of complex dimension $n$ at each point $x \in N$, it follows that $\left(\partial \rho^{1}, \ldots, \partial \rho^{2 d}\right)$ has rank $m-n=d$ at $x$.

(iii) $\Rightarrow$ (i): Since $T_{x}^{1,0} M$ is of complex dimension $m$ and $\left(\partial \rho^{1}, \ldots, \partial \rho^{2 d}\right)$ has rank $d$, the intersection of the null spaces of $\partial \rho^{\alpha}: T_{x}^{1,0} M \rightarrow \mathbb{C}, \alpha=1, \ldots, 2 d$, is of complex dimension $m-d=n$, and therefore contains linearly independent vectors $X_{1}^{1,0}, \ldots, X_{n}^{1,0}$, where $X_{k}^{1,0}=\frac{1}{2}\left(X_{k}-\sqrt{-1} J X_{k}\right)$ for some real vector $X_{k}$. Then for each $\alpha=1, \ldots, 2 d$ and each $k=1, \ldots, n$ we have, by Proposition 2.2,

$$
0=\partial \rho^{\alpha}\left(X_{k}^{1,0}\right)=\partial \rho^{\alpha}\left(X_{k}\right)=\frac{1}{2}\left(d \rho^{\alpha}\left(X_{k}\right)-\sqrt{-1} d \rho^{\alpha}\left(J X_{k}\right)\right),
$$

which implies that $d \rho^{\alpha}\left(X_{k}\right)=0$ and $d \rho^{\alpha}\left(J X_{k}\right)=0$ since the $\rho^{\alpha}$ are real-valued functions. Therefore, $\left\{X_{k}, J X_{k}: k=1, \ldots, n\right\}$ are tangent to $N$. Since $\left\{X_{k}^{1,0}, k=1, \ldots, n\right\}$ are independent, the set of vectors $X_{j}, J X_{j}(j=1, \ldots, n)$ forms a $J$-invariant basis for $T_{x} N$. Therefore, $N$ is $J$-invariant.

The $J$-invariance of submanifolds has been studied in [Han and Lee 2010]. As for the special cases of real codimension $2(d=1)$ we have the following:

Corollary 2.4. Let $(s, t)$ be a nondegenerate set of real-valued functions of $\left(M^{2 m}, J\right)$ and let $N^{2(m-1)}$ be the common zero set of $s$ and $t$. Then $N$ is $J$-invariant if and 
only if

$\partial s \wedge \partial t \equiv 0 \bmod (s, t)$.

Proof. In the case of $d=1$ in Theorem 2.3 the rank condition (iii),

$$
\operatorname{rank}(\partial s, \partial t)(x)=1 \quad \text { for each } x \in N,
$$

can be written as (2-2).

Theorem 2.5. Let $\left(M^{2 m}, J\right)$ be an almost complex manifold. A submanifold $N^{2 n}$ of real codimension $2 d$, where $d=m-n$, is $J$-invariant if and only if $N$ is the common zero set of a set of complex-valued functions $\zeta=\left(\zeta^{1}, \ldots, \zeta^{d}\right)$ that are $J$-holomorphic on the zero set.

Proof. Suppose that $N$ is a $J$-invariant submanifold of real codimension $2 d$. Let $\left(\rho^{1}, \ldots, \rho^{2 d}\right)$ be a nondegenerate set of real-valued functions whose common zero set is $N$. Since $\left(\partial \rho^{1}, \ldots, \partial \rho^{2 d}\right)$ has rank $d$ by Theorem 2.3 , we may assume that $\partial \rho^{1} \wedge \cdots \wedge \partial \rho^{d} \neq 0$. Then, for each $\alpha=1, \ldots, d, \partial \rho^{d+\alpha}$ is a linear combination of $\left(\partial \rho^{1}, \ldots, \partial \rho^{d}\right)$, or, equivalently,

$$
\left[\begin{array}{c}
\bar{\partial} \rho^{d+1} \\
\vdots \\
\bar{\partial} \rho^{2 d}
\end{array}\right]=A\left[\begin{array}{c}
\bar{\partial} \rho^{1} \\
\vdots \\
\bar{\partial} \rho^{d}
\end{array}\right]
$$

for some invertible matrix $A=\left(a_{\beta}^{\alpha}\right)$ of smooth functions. Define $\zeta=\left(\zeta^{1}, \ldots, \zeta^{d}\right)$ by

$$
\left[\begin{array}{c}
\zeta^{1} \\
\vdots \\
\zeta^{d}
\end{array}\right]=\left[\begin{array}{c}
\rho^{d+1} \\
\vdots \\
\rho^{2 d}
\end{array}\right]-A\left[\begin{array}{c}
\rho^{1} \\
\vdots \\
\rho^{d}
\end{array}\right] .
$$

By (2-3) we have

$$
\bar{\partial} \zeta^{\alpha} \equiv 0 \bmod (\rho) \text { for each } \alpha=1, \ldots, d .
$$

Since $\mathscr{I}(\rho)=\mathscr{I}(\zeta, \bar{\zeta})$ it follows that the set of complex-valued functions $\zeta=$ $\left(\zeta^{1}, \ldots, \zeta^{d}\right)$ is $J$-holomorphic on the zero set. Conversely, suppose that $\zeta=$ $\left(\zeta^{1}, \ldots, \zeta^{d}\right)$ with $d \zeta^{1} \wedge \cdots \wedge d \zeta^{d} \neq 0$ is $J$-holomorphic on the zero set. Let $\zeta^{\alpha}=s^{\alpha}+i t^{\alpha}$. Then $\bar{\partial} \zeta^{\alpha}=\bar{\partial} s^{\alpha}+i \bar{\partial} t^{\alpha} \equiv 0 \bmod (\zeta, \bar{\zeta})$, which implies

$$
\partial t^{\alpha} \equiv-i \partial s^{\alpha} \bmod (\zeta, \bar{\zeta}) .
$$

Hence the rank of $\left(\partial s^{1}, \partial t^{1}, \ldots, \partial s^{d}, \partial t^{d}\right)$ is at most $d$. On the other hand, since

$$
\begin{aligned}
d \zeta^{\alpha} & \equiv \partial \zeta^{\alpha} \quad \bmod (\zeta, \bar{\zeta}) \\
& \equiv 2 \partial s^{\alpha}, \quad \text { by }(2-4),
\end{aligned}
$$


we have

$$
\begin{aligned}
2^{d} \partial s^{1} \wedge \cdots \wedge \partial s^{d} & \equiv \partial \zeta^{1} \wedge \cdots \wedge \partial \zeta^{d} \bmod (\zeta, \bar{\zeta}) \\
& \equiv d \zeta^{1} \wedge \cdots \wedge d \zeta^{d} \bmod (\zeta, \bar{\zeta}) \\
& \neq 0
\end{aligned}
$$

Hence $\left(\partial s^{1}, \partial t^{1}, \ldots, \partial s^{d}, \partial t^{d}\right)$ has rank $d$. Then it follows from Theorem 2.3 that $N$ is $J$-invariant.

\section{Nonlinearly perturbed Cauchy-Riemann equations}

Let $z=\left(z^{1}, \ldots, z^{n}\right) \in \mathbb{C}^{n}, n \geq 1, z^{j}=x^{j}+\sqrt{-1} y^{j}$. In this section we discuss the local solvability of the quasilinear Cauchy-Riemann equations for one unknown function $w$ :

$$
\frac{\partial w}{\partial \bar{z}^{j}}+\sum_{k=1}^{n} A_{j}^{k}(z, \bar{z}, w, \bar{w}) \frac{\partial w}{\partial z^{k}}=B_{j}(z, \bar{z}, w, \bar{w}), \quad j=1, \ldots, n,
$$

where $A_{j}^{k}$ and $B_{j}$ are complex-valued $C^{\infty}$ functions defined on a neighborhood of the origin of $\mathbb{C}^{n+1}=\{(z, w)\}$.

We consider a system $\left(Z_{1}, \ldots, Z_{n+1}\right)$ of complex vector fields whose complex conjugates are given by

$$
\bar{Z}_{j}=\frac{\partial}{\partial \bar{z}^{j}}+\sum_{k=1}^{n} A_{j}^{k} \frac{\partial}{\partial z^{k}}+B_{j} \frac{\partial}{\partial w}, \quad j=1, \ldots, n, \quad \bar{Z}_{n+1}=\frac{\partial}{\partial \bar{w}} .
$$

Then $\bar{Z}_{1}, \ldots, \bar{Z}_{n+1}$ are annihilated by the following set of independent 1-forms:

$$
\theta^{\alpha}=d z^{\alpha}-\sum_{j=1}^{n} A_{j}^{\alpha} d \bar{z}^{j}, \quad \alpha=1, \ldots, n, \quad \theta^{n+1}=d w-\sum_{j=1}^{n} B_{j} d \bar{z}^{j}
$$

Let $\langle\theta\rangle$ be the linear span of $\theta=\left(\theta^{1}, \ldots, \theta^{n+1}\right)$. Then there exists the unique almost complex structure for which $\theta^{1}, \ldots, \theta^{n+1}$ are $(1,0)$ forms, provided that

$$
\langle\theta\rangle \cap\langle\bar{\theta}\rangle=\{0\},
$$

where $\bar{\theta}$ is the complex conjugate of $\theta$. Equation (3-4) holds if and only if $\theta^{1}, \ldots, \theta^{n+1}, \bar{\theta}^{1}, \ldots, \bar{\theta}^{n+1}$ are linearly independent. Note that $\theta^{1} \wedge \cdots \wedge \theta^{n+1} \wedge \bar{\theta}^{1} \wedge \cdots \wedge \bar{\theta}^{n+1}$

$$
=\left\{1+P\left(A_{j}^{\alpha}, \bar{A}_{j}^{\alpha}\right)\right\} d z^{1} \wedge d \bar{z}^{1} \wedge \cdots \wedge d z^{n} \wedge d \bar{z}^{n} \wedge d w \wedge d \bar{w},
$$

where $P$ is a polynomial in the arguments $\left(A_{j}^{\alpha}, \bar{A}_{j}^{\alpha}\right)$ without a constant term. Thus (3-4) holds if

$$
\left|P\left(A_{j}^{\alpha}, \bar{A}_{j}^{\alpha}\right)\right|<1
$$


In particular, if each $A_{j}^{\alpha}$ vanishes at the origin, then (3-5) holds. Henceforth we shall mean (3-5) by the $A_{j}^{\alpha}$ being sufficiently small.

Proposition 3.1. For each $j, k=1, \ldots, n$, let $A_{j}^{k}$ and $B_{j}$ be $C^{\infty}$ complex-valued functions defined on a neighborhood of the origin of $\mathbb{C}^{n+1}=\{(z, w)\}$. Let $J$ be the almost complex structure whose $(0,1)$-vectors and $(1,0)$-forms are given by (3-2)(3-3) assuming $A_{j}^{k}$ are sufficiently small. Suppose that $J$ has type $v$ and that $I^{(v)}$ has rank $q$. Then there exist $q$ independent $J$-holomorphic functions $\zeta^{1}, \ldots, \zeta^{q}$. A function $\zeta$ is a J-holomorphic function if and only if $\zeta$ is holomorphic in the variables $\zeta^{1}, \ldots, \zeta^{q}$.

Proof. The first part of the conclusion follows from Theorems 1.3 and 1.4. To prove the second assertion, suppose that $\zeta$ is $J$-holomorphic. Since $d \zeta \in \underline{I}^{(v)}$ we have

$$
d \zeta=\sum_{\alpha=1}^{q} a_{\alpha} d \zeta^{\alpha}
$$

for some $C^{\infty}$ functions $a_{\alpha}$. Without loss of generality assume

$$
\left(z^{1}, \ldots, z^{p}, \zeta^{1}, \ldots, \zeta^{q}\right), \quad p+q=n+1,
$$

are independent functions, so that they serve as $C^{\infty}$ local coordinates of $\mathbb{C}^{n+1}$. Then (3-6) implies

$$
\frac{\partial \zeta}{\partial z^{j}}=\frac{\partial \zeta}{\partial \bar{z}^{j}}=\frac{\partial \zeta}{\partial \bar{\zeta}^{\alpha}}=0
$$

for all $j=1, \ldots, p$ and $\alpha=1, \ldots, q$, which means that $\zeta$ is holomorphic in $\left(\zeta^{1}, \ldots, \zeta^{q}\right)$. Conversely, if $\zeta$ is a function in the variables $\zeta^{1}, \ldots, \zeta^{q}$, then we have

$$
d \zeta \in \Phi\left(d \zeta^{1}, \ldots, d \zeta^{q}\right)=I^{(v)} .
$$

Therefore, $\zeta$ is $J$-holomorphic.

Theorem 3.2. Under the same hypotheses as in Proposition 3.1, let $\zeta$ be a Jholomorphic function with $\partial \zeta / \partial w \neq 0$. Then

$$
\zeta=\text { constant }
$$

is an implicit solution of (3-1).

Proof. Since $\partial \zeta / \partial w \neq 0$ and $\partial \zeta / \partial \bar{w}=0$, by implicit function theorem we can solve (3-7) for $w$ to have $w=f(z, \bar{z})$; that is,

$$
\zeta(z, \bar{z}, f(z, \bar{z}), \overline{f(z, \bar{z})})=0 .
$$

Differentiating (3-8) in $\bar{z}^{j}$ and in $z^{k}$, respectively, we obtain

$$
\frac{\partial \zeta}{\partial \bar{z}^{j}}+\frac{\partial \zeta}{\partial w} \frac{\partial f}{\partial \bar{z}^{j}}=0, \quad \frac{\partial \zeta}{\partial z^{k}}+\frac{\partial \zeta}{\partial w} \frac{\partial f}{\partial z^{k}}=0, \quad j, k=1, \ldots, n .
$$


Since $\zeta$ is $J$-holomorphic we have $L_{j} \zeta=0, j=1, \ldots, n$; namely,

$$
\frac{\partial \zeta}{\partial \bar{z}^{j}}+\sum_{k=1}^{n} A_{j}^{k} \frac{\partial \zeta}{\partial z^{k}}+B_{j} \frac{\partial \zeta}{\partial w}=0, \quad j=1, \ldots, n .
$$

From (3-9) and (3-10) it follows that

$$
-\frac{\partial \zeta}{\partial w}\left(\frac{\partial f}{\partial \bar{z}^{j}}+\sum_{k=1}^{n} A_{j}^{k} \frac{\partial f}{\partial z^{k}}-B_{j}\right)=0
$$

which implies the conclusion.

Theorem 3.3. Let $\left(\bar{Z}_{1}, \ldots, \bar{Z}_{n+1}\right)$ and $\left(\theta^{1}, \ldots, \theta^{n+1}\right)$ be the same as in (3-2)-(3-3) and let $J$ be the almost complex structure with $(0,1)$-vectors $\bar{Z}_{j}$ (or, equivalently, $(1,0)$-forms $\left.\theta^{j}\right)$. Then there exists a solution $w=f(z, \bar{z})$ of (3-1) if and only if there exists a function $\zeta(z, \bar{z}, w, \bar{w})$ with $\partial \zeta / \partial w \neq 0$ which is $J$-holomorphic on the zero set.

Proof. Suppose that $w=f(z, \bar{z})$ is a solution of (3-1). Then

$$
\zeta(z, \bar{z}, w, \bar{w}):=f(z, \bar{z})-w
$$

satisfies $\bar{Z}_{j} \zeta \equiv 0, \bmod (\zeta, \bar{\zeta})$, for all $j=1, \ldots, n+1$. Conversely, suppose that $\zeta(z, \bar{z}, w, \bar{w})$ with $\partial \zeta / \partial w \neq 0$ is $J$-holomorphic on the zero set. Since $\partial \zeta / \partial \bar{w}=0$ on the zero set, by the implicit function theorem we can solve $\zeta=0$ for $w$, to obtain $w=f(z, \bar{z})$; that is,

$$
\zeta(z, \bar{z}, f(z, \bar{z}), \overline{f(z, \bar{z})})=0 .
$$

Then by differentiating (3-12) with respect to $\bar{z}^{j}$ and $z^{k}$ and restricting to the zero set of $\zeta$ we have (3-9)-(3-11) and the proof is same as that of Theorem 3.2.

For the existence of solutions of (3-1) the coefficients $A_{j}^{k}$ and $B_{j}$ must satisfy certain conditions. To discuss this we first define smooth functions $T_{i j}^{\alpha}$ by setting

$$
d \theta^{\alpha} \equiv \sum_{1 \leq i<j \leq n+1} T_{i j}^{\alpha} d \bar{z}^{i} \wedge d \bar{z}^{j} \bmod (\theta), \quad \alpha=1, \ldots, n+1,
$$

where $z^{n+1}=w, \bar{z}^{n+1}=\bar{w}$. Arranging the pairs $(i j)$ with $i<j$ in lexicographical order, we write (3-13) in matrices as

$$
\left[\begin{array}{c}
d \theta^{1} \\
\vdots \\
d \theta^{n+1}
\end{array}\right] \equiv \underbrace{\left[\begin{array}{cccc}
T_{12}^{1} & T_{13}^{1} & \cdots & T_{n, n+1}^{1} \\
\vdots & \vdots & & \vdots \\
T_{12}^{n+1} & T_{13}^{n+1} & \cdots & T_{n, n+1}^{n+1}
\end{array}\right]}_{\mathcal{T}}\left[\begin{array}{c}
d \bar{z}^{1} \wedge d \bar{z}^{2} \\
d \bar{z}^{1} \wedge d \bar{z}^{3} \\
\vdots \\
d \bar{z}^{n} \wedge d \bar{z}^{n+1}
\end{array}\right]
$$

$\bmod (\theta)$. 
The matrix $\mathscr{T}$ of size $(n+1) \times\left(\begin{array}{c}n+1 \\ 2\end{array}\right)$ shall be called the torsion of the Pfaffian system (3-3).

If $\mathcal{T}$ has rank zero, that is, if all $T_{i j}^{\alpha}$ are zero, this is the case $I=I^{(1)}$, and $I$ is closed. Then by Theorem 1.2 there exist $n+1$ independent $J$-holomorphic functions.

Theorem 3.4. Suppose there exist $J$-holomorphic functions $\zeta^{1}, \ldots, \zeta^{q}, q \leq n+1$, with $d \zeta^{1} \wedge \cdots \wedge d \zeta^{q} \neq 0$. Then $\mathcal{T}$ has rank at most $(n+1)-q$.

Proof. For each $\lambda=1, \ldots, q$, let

$$
d \zeta^{\lambda}=\sum_{\alpha=1}^{n+1} a_{\alpha}^{\lambda} \theta^{\alpha}
$$

for some functions $a_{\alpha}^{\lambda}$. Applying $d$ to (3-14) we have

$$
0 \equiv \sum_{\alpha} a_{\alpha}^{\lambda} d \theta^{\alpha} \bmod (\theta)
$$

for each $\lambda=1, \ldots, q$. Substituting (3-13) in (3-15) we have

$$
0 \equiv \sum_{\alpha=1}^{n+1} a_{\alpha}^{\lambda} \sum_{1 \leq i<j \leq n+1} T_{i j}^{\alpha} d \bar{z}^{i} \wedge d \bar{z}^{j} \bmod (\theta),
$$

which is written in matrices as

$$
\left[\begin{array}{c}
0 \\
\vdots \\
0
\end{array}\right] \equiv \underbrace{\left[\begin{array}{ccc}
a_{1}^{1} & \cdots & a_{n+1}^{1} \\
\vdots & & \vdots \\
a_{1}^{q} & \cdots & a_{n+1}^{q}
\end{array}\right]}_{\mathcal{A}} \underbrace{\left[\begin{array}{cccc}
T_{12}^{1} & T_{13}^{1} & \cdots & T_{n, n+1}^{1} \\
\vdots & \vdots & & \vdots \\
T_{12}^{n+1} & T_{13}^{n+1} & \cdots & T_{n, n+1}^{n+1}
\end{array}\right]}_{\mathcal{T}}\left[\begin{array}{c}
d \bar{z}^{1} \wedge d \bar{z}^{2} \\
d \bar{z}^{1} \wedge d \bar{z}^{3} \\
\vdots \\
d \bar{z}^{n} \wedge d \bar{z}^{n+1}
\end{array}\right] \bmod (\theta) .
$$

Since $d \zeta^{1} \wedge \cdots \wedge d \zeta^{q} \neq 0,(3-14)$ implies that $\mathscr{A}$ has maximal rank $q$. Each row of $\mathscr{A}$ gives a linear relation among the rows of $\mathscr{T}$. Therefore, $\mathscr{T}$ has rank at most $(n+1)-q$.

We construct the sequence (1-4) of subbundles as follows: an element $\phi=$ $\sum_{\alpha=1}^{n+1} a_{\alpha} \theta^{\alpha}$ of $I$ belongs to $I^{(1)}$ if and only if $\left(a_{1}, \ldots, a_{n+1}\right)$ is a null vector of the matrix $\mathscr{T}$, because

$$
\begin{aligned}
d \phi & \equiv \sum_{\alpha=1}^{n+1} a_{\alpha} d \theta^{\alpha} \bmod (\theta) \\
& \equiv \sum_{1 \leq i<j \leq n+1} \sum_{\alpha=1}^{n+1} a_{\alpha} T_{i j}^{\alpha} d \bar{z}^{i} \wedge d \bar{z}^{j} \bmod (\theta)
\end{aligned}
$$


is zero if and only if $\left(a_{1}, \ldots, a_{n+1}\right)$ is a null vector of $\mathscr{T}$; that is,

$$
\sum_{\alpha=1}^{n+1} a_{\alpha} T_{i j}^{\alpha}=0 \quad \text { for all pairs }(i j) \text {. }
$$

Inductively, let $\phi=\left(\phi^{1}, \ldots, \phi^{r}\right)$ be a set of generators of $I^{(k)}$. Then a 1 -form $\psi=\sum_{\beta=1}^{r} b_{\beta} \phi^{\beta}$ is an element of $I^{(k+1)}$ if and only if $\left(b_{1}, \ldots, b_{r}\right)$ is a null vector of the torsion matrix of the Pfaffian system $\phi$. Now suppose that (3-3) has derived length $v$. In the construction of $I^{(v)}$ the coefficients $A_{j}^{k}, B_{j}$ in (3-3) are differentiated up to $v$ times and then the condition

$$
d I^{(v)} \subset\left(I^{(v)}\right)
$$

raises the order of the derivatives by one. Thus we have:

Proposition 3.5. Let $J$ be the almost complex structure on $\mathbb{C}^{n+1}$ whose $(1,0)$-forms are given in (3-3). Then its type condition is a system of partial differential equations on $\left(A_{j}^{k}, B_{j}\right)$ : condition (3-16) being of type $v$ is a PDE system of order $v+1$. If $J$ has type $v$ and $I^{(v)}$ has rank $q$, then there exists a complex $q$-parameter family of solutions of (3-1).

Summarizing our previous discussions in Theorems 3.3 and 2.5 and Corollary 2.4 we have:

Theorem 3.6. Given a system of quasilinear Cauchy-Riemann equations (3-1) with coefficients $A_{j}^{\alpha}$ sufficiently small, let $J$ be the almost complex structure on $\mathbb{C}^{n+1}=\{(z, w)\}, z=\left(z^{1}, \ldots, z^{n}\right), n \geq 2$, with (1,0)-forms (3-3). Then (3-1) has a solution if and only if there exists a nondegenerate system of real-valued functions $(s, t)$ having the following properties:

(i) The determinant of any square submatrices of maximal size of $\mathcal{T}$ is zero modulo $(s, t)$.

(ii) $\partial s \wedge \partial t \equiv 0 \bmod (s, t)$.

Condition (ii) means that the common zero set of $s$ and $t$ is $J$-invariant. Condition (i) means that we construct $s$ and $t$ by finding a nondegenerate set of real-valued functions that generates an ideal to which the determinants of $n \times n$ submatrices of the torsion belong.

\section{Cases of several unknown functions}

Our arguments of the previous section can easily be generalized to the cases of several unknown functions. Let $z=\left(z^{1}, \ldots, z^{n}\right) \in \mathbb{C}^{n}, n \geq 1, z^{j}=x^{j}+\sqrt{-1} y^{j}$. We consider the system of quasilinear Cauchy-Riemann equations for $w=\left(w^{1}, \ldots, w^{d}\right)$, 
$d \geq 2:$

$$
\frac{\partial w^{\alpha}}{\partial \bar{z}^{j}}+\sum_{k=1}^{n} A_{j}^{k}(z, \bar{z}, w, \bar{w}) \frac{\partial w^{\alpha}}{\partial z^{k}}=B_{j}^{\alpha}(z, \bar{z}, w, \bar{w})
$$

for each $j=1, \ldots, n$ and $\alpha=1, \ldots, d$, where $A_{j}^{k}$ and $B_{j}^{\alpha}$ are complex-valued $C^{\infty}$ functions defined on a neighborhood of the origin of $\mathbb{C}^{n+d}=\{(z, w)\}$. We consider a system $\left(Z_{1}, \ldots, Z_{n+d}\right)$ of complex vector fields on an open neighborhood of the origin of $\mathbb{C}^{n+d}=\{(z, w)\}$ whose complex conjugates are given by

$$
\begin{gathered}
\bar{Z}_{j}=\frac{\partial}{\partial \bar{z}^{j}}+\sum_{k=1}^{n} A_{j}^{k} \frac{\partial}{\partial z^{k}}+\sum_{\alpha=1}^{d} B_{j}^{\alpha} \frac{\partial}{\partial w^{\alpha}}, \quad j=1, \ldots, n, \\
\bar{Z}_{n+\beta}=\frac{\partial}{\partial \bar{w}^{\beta}}, \quad \beta=1, \ldots, d .
\end{gathered}
$$

Then $\bar{Z}_{1}, \ldots, \bar{Z}_{n+d}$ are annihilated by the following set of independent 1-forms:

$$
\begin{gathered}
\theta^{k}=d z^{k}-\sum_{j=1}^{n} A_{j}^{k} d \bar{z}^{j}, \quad k=1, \ldots, n, \\
\theta^{n+\alpha}=d w^{\alpha}-\sum_{j=1}^{n} B_{j}^{\alpha} d \bar{z}^{j}, \quad \alpha=1, \ldots, d .
\end{gathered}
$$

If $A_{j}^{k}$ are sufficiently small, then the functions $A_{j}^{k}$ and $B_{j}^{\alpha}$ define an almost complex structure $J$ on $\mathbb{C}^{n+d}$, for which $\bar{Z}_{j}, \bar{Z}_{n+\beta}$ are $(0,1)$-vector fields, or, equivalently, $\theta^{k}, \theta^{n+\alpha}$ are (1,0)-forms. The following is a generalization of Theorem 3.3.

Theorem 4.1. Let $\left(\bar{Z}_{1}, \ldots, \bar{Z}_{n+d}\right)$ and $\left(\theta^{1}, \ldots, \theta^{n+d}\right)$ be the same as in (4-2)(4-3) and let $J$ be the almost complex structure with $(0,1)$-vectors $\bar{Z}_{j}$ (or, equivalently, (1,0)-forms $\left.\theta^{j}\right)$. Then there exists a set of solutions $w^{\alpha}=f^{\alpha}(z, \bar{z})$, $\alpha=1, \ldots, d$, of (4-1) if and only if there exists a set of functions $\zeta^{\alpha}(z, \bar{z}, w, \bar{w})$, $\alpha=1, \ldots, d$ with $\operatorname{det}\left(\partial \zeta^{\alpha} / \partial w^{\beta}\right) \neq 0$ that is $J$-holomorphic on the zero set.

Proof. Suppose $w^{\alpha}=f^{\alpha}(z, \bar{z}), \alpha=1, \ldots, d$, is a solution of (4-1). Let $\zeta^{\alpha}=$ $f^{\alpha}(z, \bar{z})-w^{\alpha}$. Then, for each $j=1, \ldots, n$ and each $\alpha=1, \ldots, d$, we have

$$
\bar{Z}_{j} \zeta^{\alpha}=\frac{\partial f^{\alpha}}{\partial \bar{z}^{j}}+A_{j}^{k} \frac{\partial f^{\alpha}}{\partial z^{k}}-B_{j}^{\alpha} \equiv 0 \bmod (\zeta, \bar{\zeta}), \quad \bar{Z}_{n+\beta} \zeta^{\alpha}=0
$$

Therefore, $\zeta=\left(\zeta^{1}, \ldots, \zeta^{d}\right)$ is $J$-holomorphic on the zero set that satisfies the nondegeneracy condition as in the statement of the theorem. Conversely, suppose that $\zeta=\left(\zeta^{1}, \ldots, \zeta^{d}\right)$ is $J$-holomorphic on the zero set as in the statement of the theorem. Since $\operatorname{det}\left(\partial \zeta^{\alpha} / \partial w^{\beta}\right) \neq 0$ and $\partial \zeta^{\alpha} / \partial \bar{w}^{\beta}=0 \bmod (\zeta, \bar{\zeta})$, we can solve 
$\zeta=0$ for $w=\left(w^{1}, \ldots, w^{d}\right)$ by implicit function theorem, to obtain $w^{\alpha}=f^{\alpha}(z, \bar{z})$; that is,

$$
\zeta^{\alpha}(z, \bar{z}, f(z, \bar{z}), \overline{f(z, \bar{z})})=0, \quad \alpha=1, \ldots, d,
$$

where $f=\left(f^{1}, \ldots, f^{d}\right)$. By applying $\partial / \partial \bar{z}^{j}$ and $\partial / \partial z^{k}$, respectively, to (4-4) we have

$$
\frac{\partial \zeta^{\alpha}}{\partial \bar{z}^{j}}+\sum_{\beta=1}^{d} \frac{\partial \zeta^{\alpha}}{\partial w^{\beta}} \frac{\partial f^{\beta}}{\partial \bar{z}^{j}}=0, \quad \frac{\partial \zeta^{\alpha}}{\partial z^{k}}+\sum_{\beta=1}^{d} \frac{\partial \zeta^{\alpha}}{\partial w^{\beta}} \frac{\partial f^{\beta}}{\partial z^{k}}=0 .
$$

Then, for each $j=1, \ldots, n$ and each $\alpha=1, \ldots, d$, we have

$$
\begin{aligned}
\bar{Z}_{j} \zeta^{\alpha} & =\frac{\partial \zeta^{\alpha}}{\partial \bar{z}^{j}}+\sum_{k=1}^{n} A_{j}^{k}(z, \bar{z}, w, \bar{w}) \frac{\partial \zeta^{\alpha}}{\partial z^{k}}+\sum_{\beta=1}^{d} B_{j}^{\beta}(z, \bar{z}, w, \bar{w}) \frac{\partial \zeta^{\alpha}}{\partial w^{\beta}} \\
& \equiv 0 \bmod (\zeta, \bar{\zeta}) .
\end{aligned}
$$

Combining (4-5) and (4-6) we have

$$
-\sum_{\beta=1}^{d} \frac{\partial \zeta^{\alpha}}{\partial w^{\beta}} \frac{\partial f^{\beta}}{\partial \bar{z}^{j}}-\sum_{k=1}^{n} \sum_{\beta=1}^{d} A_{j}^{k} \frac{\partial \zeta^{\alpha}}{\partial w^{\beta}} \frac{\partial f^{\beta}}{\partial z^{k}}+\sum_{\beta=1}^{d} B_{j}^{\beta} \frac{\partial \zeta^{\alpha}}{\partial w^{\beta}} \equiv 0 \bmod (\zeta, \bar{\zeta}),
$$

which can be written in matrices as

$$
\frac{\partial \zeta}{\partial w} E \equiv\left[\begin{array}{ccc}
0 & \cdots & 0 \\
\vdots & \ddots & \vdots \\
0 & \cdots & 0
\end{array}\right]_{d \times n} \bmod (\zeta, \bar{\zeta})
$$

where

$$
E=\left[\begin{array}{ccc}
\frac{\partial \zeta}{\partial w}=\left[\begin{array}{ccc}
\frac{\partial \zeta^{1}}{\partial w^{1}} & \cdots & \frac{\partial \zeta^{1}}{\partial w^{d}} \\
\vdots & \ddots & \vdots \\
\frac{\partial \zeta^{d}}{\partial w^{1}} & \cdots & \frac{\partial \zeta^{d}}{\partial w^{d}}
\end{array}\right], \\
-\frac{\partial f^{1}}{\partial \bar{z}^{1}}-\sum_{k=1}^{n} A_{1}^{k} \frac{\partial f^{1}}{\partial z^{k}}+B_{1}^{1} & \cdots & -\frac{\partial f^{1}}{\partial \bar{z}^{n}}-\sum_{k=1}^{n} A_{n}^{k} \frac{\partial f^{1}}{\partial z^{k}}+B_{n}^{1} \\
\vdots & \ddots & \vdots \\
-\frac{\partial f^{d}}{\partial \bar{z}^{1}}-\sum_{k=1}^{n} A_{1}^{k} \frac{\partial f^{d}}{\partial z^{k}}+B_{1}^{d} & \cdots & -\frac{\partial f^{n}}{\partial \bar{z}^{n}}-\sum_{k=1}^{n} A_{n}^{k} \frac{\partial f^{d}}{\partial z^{k}}+B_{n}^{d}
\end{array}\right]_{d \times n} .
$$

Since $\partial \zeta / \partial w$ is invertible $E$ is identically zero on the zero set of $\zeta$, which implies that $w=f(z, \bar{z})$ is a solution of (4-1). 
Then the almost complex structure on $\mathbb{C}^{n+d}$ whose $(0,1)$-forms are given by (4-2) has torsion $\mathcal{T}$ of dimension $(n+d) \times\left(\begin{array}{c}n+d \\ 2\end{array}\right)$. By the same argument as in the previous section we have:

Theorem 4.2. Given a system of quasilinear Cauchy-Riemann equations (4-1), let $J$ be the almost complex structure on $\mathbb{C}^{n+d}=\{(z, w)\}, z=\left(z^{1}, \ldots, z^{n}\right)$, $w=\left(w^{1}, \ldots, w^{d}\right)$, with $(1,0)$-forms (4-3). Then (4-1) has a solution if and only if there exists a nondegenerate system of real-valued functions $\left(s^{1}, \ldots, s^{2 d}\right)$ having the following properties:

(i) The determinant of any $n \times n$ submatrix of the $(n+d) \times\left(\begin{array}{c}n+d \\ 2\end{array}\right)$ matrix of the torsion $\mathcal{T}$ is zero modulo $\left(s^{1}, \ldots, s^{2 d}\right)$.

(ii) $\left(\partial s^{1}, \ldots, \partial s^{2 d}\right)$ has rank $d$.

Condition (ii) means that the common zero set of $s^{1}, \ldots, s^{2 d}$ is $J$-invariant. Condition (i) means that we construct $s^{1}, \ldots, s^{2 d}$ by finding a nondegenerate set of real-valued functions that generates an ideal to which the determinants of $n \times n$ submatrices of the torsion belong.

\section{Quasilinear Cauchy-Riemann equations in one complex variable}

Consider the following equation for a complex-valued function $w=w(z, \bar{z})$ :

$$
\frac{\partial w}{\partial \bar{z}}+A(z, \bar{z}, w, \bar{w}) \frac{\partial w}{\partial z}=B(z, \bar{z}, w, \bar{w}), \quad|A(z, \bar{z}, w, \bar{w})|<1 .
$$

This is a determined system of two real equations for two real unknown functions $\Re w$ and $\Im w$. Equation (5-1) is always solvable for the following reason: in $\mathbb{C}^{2}=\{(z, w)\}$ we consider complex vector fields

$$
\bar{Z}_{1}=\frac{\partial}{\partial \bar{z}}+A \frac{\partial}{\partial z}+B \frac{\partial}{\partial w}, \quad \bar{Z}_{2}=\frac{\partial}{\partial \bar{w}}
$$

and 1-forms that annihilate $\bar{Z}_{j}, j=1,2$ :

$$
\theta^{1}=d z-A d \bar{z}, \quad \theta^{2}=d w-B d \bar{z} .
$$

An almost complex structure $J$ on $\mathbb{C}^{2}$ is uniquely determined by the functions $A$ and $B$ so that $\bar{Z}_{j}, j=1,2$, are $(0,1)$-vectors and $\theta^{j}, j=1,2$, are $(1,0)$-forms. A fundamental theorem of [Nijenhuis and Woolf 1963] states that for any real tangent vector $V$ of $\mathbb{C}^{2}$ at the origin there exists a $J$-holomorphic curve $\gamma(z)=(z, f(z))$ : $D \rightarrow \mathbb{C}^{2}$, where $D$ is a small open disk centered at the origin in $\mathbb{C}$, satisfying initial conditions $\gamma(0)=0$ and $d \gamma(0)(\partial / \partial x)=V$. The graph $\gamma$ is the zero set of

$$
\zeta:=f(z, \bar{z})-w,
$$

which is $J$-holomorphic on its zero set. Thus (5-1) is solvable by Theorem 3.3. 
Now we check the type of $J$. Since

$$
\left[\begin{array}{l}
d \theta^{1} \\
d \theta^{2}
\end{array}\right] \equiv \mathscr{T} d \bar{z} \wedge d \bar{w} \bmod (\theta), \quad \text { where } \mathscr{T}=\left[\begin{array}{l}
A_{\bar{w}} \\
B_{\bar{w}}
\end{array}\right],
$$

if $A_{\bar{w}}=B_{\bar{w}}=0$ the almost complex structure is integrable, and hence by Theorem 1.2 there exist two independent $J$-holomorphic functions $\left(\zeta^{1}, \zeta^{2}\right)$. For any function $\zeta$ that is analytic in $\left(\zeta^{1}, \zeta^{2}\right)$ such that $\zeta_{w} \neq 0$,

$$
\zeta=\text { constant }
$$

is an implicit solution of (5-1). This is the case of type 0 . Next, we assume $A_{\bar{w}} \neq 0$. Then $\left(-B_{\bar{w}}, A_{\bar{w}}\right)$ is a null vector of the torsion $\mathscr{T}$, so that

$$
\phi:=-B_{\bar{w}} \theta^{1}+A_{\bar{w}} \theta^{2}
$$

generates $I^{(1)}$. If

$$
d \phi \equiv 0 \bmod \phi
$$

then $I^{(1)}$ is closed. Equation (5-3) is a PDE system of second order for $A$ and $B$. In summary we have the following table:

\begin{tabular}{|ccccc|}
\hline rank $\mathscr{T}$ & type $v$ & $\begin{array}{c}\text { number of } \\
J \text {-holomorphic } \\
\text { functions }\end{array}$ & $\begin{array}{c}\text { order of PDE } \\
\text { for } A, B\end{array}$ & integrability \\
\hline 0 & 0 & 2 & 1 & integrable \\
1 & 1 & 1 & 2 & $I^{(1)}$ is closed \\
\hline
\end{tabular}

Let us consider the following special case of type 1:

$$
\frac{\partial w}{\partial \bar{z}}+A(z, \bar{z}, w, \bar{w}) \frac{\partial w}{\partial z}=B(\bar{z}, w), \quad A_{\bar{w}} \neq 0 .
$$

Since $B_{\bar{w}}=0$, from (5-2), $\phi=A_{\bar{w}} \theta^{2}$ generates $I^{(1)}$. Then computation shows

$$
d \phi \equiv 0 \bmod (\phi) .
$$

Thus $I^{(1)}$ has rank 1 and there is a nondegenerate $J$-holomorphic function $\zeta$. Since $d \zeta \in \mathscr{I}\left(\theta^{2}\right)$ we see that $\zeta_{w} \neq 0$. Therefore,

$$
\zeta=\text { constant }
$$

is a complex 1-parameter family of solutions of (5-4). 


\section{Examples}

Example 6.1. Consider the following system for $w\left(z^{1}, \bar{z}^{1}, z^{2}, \bar{z}^{2}\right)$ :

$$
\begin{aligned}
& \frac{\partial w}{\partial \bar{z}^{1}} \quad+w \frac{\partial w}{\partial z^{2}}=\frac{-2 w}{1+\bar{z}^{1}}, \\
& \frac{\partial w}{\partial \bar{z}^{2}}+\bar{w} \frac{\partial w}{\partial z^{1}}+\bar{z}^{1} \frac{\partial w}{\partial z^{2}}=0 .
\end{aligned}
$$

Then the associated almost complex structure on $\mathbb{C}^{3}=\left\{\left(z^{1}, z^{2}, w\right)\right\}$ has $(1,0)$ forms

$$
\begin{array}{ll}
\theta^{1} & =d z^{1} \quad-\bar{w} d \bar{z}^{2}, \\
\theta^{2} & =d z^{2} \quad-w d \bar{z}^{1}-\bar{z}^{1} d \bar{z}^{2}, \\
\theta^{3} & =d w+\frac{2 w}{1+\bar{z}^{1}} d \bar{z}^{1} .
\end{array}
$$

Then we have

$$
\left[\begin{array}{l}
d \theta^{1} \\
d \theta^{2} \\
d \theta^{3}
\end{array}\right]=\underbrace{\left[\begin{array}{ccc}
0 & 0 & 1 \\
-1 & 0 & 0 \\
0 & 0 & 0
\end{array}\right]}_{\mathcal{T}}\left[\begin{array}{l}
d \bar{z}^{1} \wedge d \bar{z}^{2} \\
d \bar{z}^{1} \wedge d \bar{w} \\
d \bar{z}^{2} \wedge d \bar{w}
\end{array}\right] \bmod (\theta) .
$$

Hence, $I^{(1)}$ is spanned by $\theta^{3}$. Since

$$
d \theta^{3} \equiv 0 \bmod \left(\theta^{3}\right)
$$

this is the case of type 1 . There exists a $J$-holomorphic function $\zeta$. Since $d \zeta$ is a nonzero multiple of $\theta^{3}$ we see that $\zeta_{w} \neq 0$. Each level set $\zeta=$ constant is an implicit solution of (6-1).

Example 6.2. Consider the following system for $w\left(z^{1}, \bar{z}^{1}, z^{2}, \bar{z}^{2}\right)$ :

$$
\begin{aligned}
\frac{\partial w}{\partial \bar{z}^{1}}+w \frac{\partial w}{\partial z^{2}} & =\frac{-2 w}{1+\bar{z}^{1}}, \\
\frac{\partial w}{\partial \bar{z}^{2}}+\bar{w} \frac{\partial w}{\partial z^{1}}+\left[z^{2}+w\left(1+\bar{z}^{1}\right)\right] \frac{\partial w}{\partial z^{2}} & =0 .
\end{aligned}
$$

Then the associated almost complex structure on $\mathbb{C}^{3}=\left\{\left(z^{1}, z^{2}, w\right)\right\}$ has $(1,0)$ forms

$$
\begin{array}{ll}
\theta^{1} & =d z^{1} \\
\theta^{2} & =d z^{2} \quad-\bar{w} d \bar{z}^{2}, \\
\theta^{3} & =d w+\frac{2 w}{1+\bar{z}^{1}} d \bar{z}^{1} .
\end{array}
$$


Let

$$
\zeta:=z^{2}+w\left(1+\bar{z}^{1}\right)
$$

Then we see that

$$
d \zeta=\theta^{2}+\left(1+\bar{z}^{1}\right) \theta^{3}+\zeta d \bar{z}^{2} .
$$

Therefore, $\zeta$ is $J$-holomorphic on its zero set. Thus $\zeta=0$ is an implicit solution of (6-2).

A pseudoanalytic function in several complex variables satisfies

$$
\frac{\partial w}{\partial \bar{z}^{j}}=\alpha_{j}(z) w(z)+\beta_{j}(z) \overline{w(z)} \text { for } j=1, \ldots, n,
$$

for some functions $\alpha_{j}(z)$ and $\beta_{j}(z)$. See the details in Section 7.

Example 6.3. Consider the system (7-4) of pseudoanalytic functions in $\mathbb{C}^{2}$ :

$$
\frac{\partial w}{\partial \bar{z}_{1}}=\alpha_{1}(z, \bar{z}) w+\beta_{1}(z, \bar{z}) \bar{w}, \quad \frac{\partial w}{\partial \bar{z}_{2}}=\alpha_{2}(z, \bar{z}) w+\beta_{2}(z, \bar{z}) \bar{w} .
$$

Let $B_{j}(z, \bar{z}, w, \bar{w})=\alpha_{j}(z, \bar{z}) w+\beta_{j}(z, \bar{z}) \bar{w}$ for $j=1,2$. Then the associated almost complex structure on $\mathbb{C}^{3}=\left\{\left(z^{1}, z^{2}, w\right)\right\}$ has $(1,0)$-forms

$$
\theta^{1}=d z^{1}, \quad \theta^{2}=d z^{2}, \quad \theta^{3}=d w-B_{1} d \bar{z}^{1}-B_{2} d \bar{z}^{2} .
$$

By applying $d$ to $\theta^{1}, \theta^{2}, \theta^{3}$, we obtain the components of the torsion $\mathcal{T}$ as follows:

$$
\begin{aligned}
T_{i j}^{\alpha} & =0 \quad \text { for } \alpha=1,2, \\
T_{12}^{3} & =\frac{\partial B_{1}}{\partial \bar{z}^{2}}+\frac{\partial B_{1}}{\partial w} B_{2}-\frac{\partial B_{2}}{\partial \bar{z}^{1}}-\frac{\partial B_{2}}{\partial w} B_{1} \\
& =\left(\frac{\partial \alpha_{1}}{\partial \bar{z}^{2}}-\frac{\partial \alpha_{2}}{\partial \bar{z}^{1}}\right) w+\left(\frac{\partial \beta_{1}}{\partial \bar{z}^{2}}-\frac{\partial \beta_{2}}{\partial \bar{z}^{1}}+\alpha_{1} \beta_{2}-\alpha_{2} \beta_{1}\right) \bar{w}, \\
T_{13}^{3} & =\frac{\partial B_{1}}{\partial \bar{w}}=\beta_{1}, \quad T_{23}^{3}=\frac{\partial B_{2}}{\partial \bar{w}}=\beta_{2} .
\end{aligned}
$$

Then $\mathscr{T}$ has rank 0 if and only if $\beta_{1}=\beta_{2}=0$ and

$$
\frac{\partial \alpha_{1}}{\partial \bar{z}^{2}}=\frac{\partial \alpha_{2}}{\partial \bar{z}^{1}} .
$$

This is the involutive case and there exist three independent pseudoholomorphic functions for the associated almost complex structure. One of them satisfies $\partial \zeta / \partial w \neq 0$, which gives implicit solutions $\zeta=$ constant.

If rank $\mathscr{T}=1$, then $\phi^{1}=\theta^{1}$ and $\phi^{2}=\theta^{2}$ generates $I^{(1)}$. Since $d \phi^{k}=0$ for $k=1,2$, this is the case of type 2 . However, it is easy to check that there cannot be a function $\zeta$ with $\partial \zeta / \partial w \neq 0$ that is pseudoholomorphic on the zero set. Therefore, there are no solutions in this case. 


\section{Perturbed Cauchy-Riemann equations and overdetermined PDE systems: a brief survey of history}

The main object of complex analysis is the family of holomorphic functions $w=w(z)$ which are characterized by the Cauchy-Riemann equations

$$
\frac{\partial w}{\partial \bar{z}^{j}}=0, \quad j=1, \ldots, n .
$$

It is not surprising that mathematical literature abounds in natural generalizations of the Cauchy-Riemann equations. For the cases $n=1$ the theories of quasiconformal functions and pseudoanalytic functions were developed in the mid-20th century [Ahlfors 1954; 1966; Bers 1956; 1977]. A quasiconformal mapping $w=w(z)$ satisfies the Beltrami equation

$$
\frac{\partial w}{\partial \bar{z}}+\mu(z) \frac{\partial w}{\partial z}=0
$$

for some complex-valued Lebesgue measurable function $\mu(z)$ with $|\mu(z)|<1$. Of central importance in the theory of quasiconformal mappings in the complex plane is the measurable Riemann mapping theorem [Morrey 1938], which generalizes the Riemann mapping theorem from conformal to quasiconformal homeomorphisms.

Pseudoanalytic functions [Bers 1953; Vekua 1962] are solutions of

$$
\frac{\partial w}{\partial \bar{z}}=\alpha(z) w(z)+\beta(z) \overline{w(z)}
$$

for some functions $\alpha(z)$ and $\beta(z)$. Recall that every harmonic function $\phi(x, y)$ is locally the real part of an analytic function $h(z)$ and the complex gradient $w(z)=\partial \phi / \partial x-i \partial \phi / \partial y$ is analytic and $w(z)=h^{\prime}(z)$. On the other hand, a linear elliptic equation for a real-valued function $\phi(x, y)$ of second order with Hölder continuously differentiable coefficients can be transformed into the canonical form

$$
\phi_{x x}+\phi_{y y}+A \phi_{x}+B \phi_{y}=0 .
$$

Then $w:=\partial \phi / \partial x-i \partial \phi / \partial y$ is a pseudoanalytic function which satisfies (7-2) with $\alpha=-\frac{1}{4}(A+i B)$ and $\beta=-\frac{1}{4}(A-i B)$.

In $\mathbb{C}^{n}$ with $n \geq 2$, fundamental questions including the Levi problem were solved by means of the inhomogeneous Cauchy-Riemann equations of $(p, q)$-type (see [Oka 1953; Kohn 1963; Hörmander 1965]), which are generalizations of the $(0,1)$-type equations

$$
\frac{\partial w}{\partial \bar{z}^{j}}=b_{j} \quad \text { for } j=1, \ldots, n \text {. }
$$


A multidimensional version of (7-1) is

$$
\boldsymbol{L}_{j} w:=\frac{\partial w}{\partial \bar{z}^{j}}(z)+\sum_{k=1}^{n} a_{j}^{k}(z) \frac{\partial w}{\partial z^{k}}(z)=0 \quad \text { for } j=1, \ldots, n,
$$

where the $a_{j}^{k}$ vanish at the origin. If the coefficients $a_{j}^{k}(z)$ are sufficiently smooth, it was shown in [Newlander and Nirenberg 1957] that there exist $n$ linearly independent solutions to (7-3) if and only if $\boldsymbol{L}_{j}$ commutes. We state this fundamental result as Theorem 1.2.

A pseudoanalytic function in several complex variables satisfies

$$
\frac{\partial w}{\partial \bar{z}^{j}}=\alpha_{j}(z) w(z)+\beta_{j}(z) \overline{w(z)} \quad \text { for } j=1, \ldots, n,
$$

for some functions $\alpha_{j}(z)$ and $\beta_{j}(z)$. The properties of solutions to (7-4) were investigated in [So'n 1990; Hayashi 1996]. In particular, it was proved in [So'n 1990] that (7-4) with $\beta_{j}(z)=0$ has the extension property: if $D \subset \mathbb{C}^{n}$ is a domain and $K$ is a compact subset of $D$ such that $D \backslash K$ is connected, then any solution to (7-4) with $\beta_{j}(z)=0$ on $D \backslash K$ extends to $D$. The extension phenomenon of holomorphic functions in several complex variables is the special case of this extension property for (7-4) with $\alpha_{j}(z)=\beta_{j}(z)=0$, which was discovered in [Hartogs 1906].

In the 1960s, the theory of overdetermined systems of linear partial differential equations was intensively studied from the algebraic viewpoint based on Spencer complexes [1962; 1965; 1969]. Quillen [1964], Goldschmidt [1967], MacKichan [1968] and Sweeney [1968] investigated the condition for the exactness of the Spencer complexes. The question was whether the Spencer complex is exact if it is elliptic. When the coefficients are real analytic the Spencer complex is exact in the elliptic case. In the $C^{\infty}$ category, however, one needs to prove an estimate that implies the solvability of the associated Neumann boundary value problem. It turned out that, if the linear elliptic overdetermined system satisfies the so-called $\delta$-estimate, then the Neumann problem for the elliptic system is solvable so that the Spencer sequence is exact and hence such a system is locally solvable.

\section{References}

[Ahlfors 1954] L. V. Ahlfors, "On quasiconformal mappings", J. Analyse Math. 3 (1954), 1-58. MR 16,348d Zbl 0057.06506

[Ahlfors 1966] L. V. Ahlfors, Lectures on quasiconformal mappings, Van Nostrand Mathematical Studies 10, D. Van Nostrand, Princeton, 1966. MR 34 \#336 Zbl 0138.06002

[Ahn and Han 2012] H. Ahn and C.-K. Han, "Local geometry of Levi-forms associated with the existence of complex submanifolds and the minimality of generic CR manifolds", J. Geom. Anal. 22:2 (2012), 561-582. MR 2891737 Zbl 06112517 
[Berhanu et al. 2008] S. Berhanu, P. D. Cordaro, and J. Hounie, An introduction to involutive structures, New Mathematical Monographs 6, Cambridge University Press, 2008. MR 2009b:32048 Zbl 1151.35011

[Bers 1953] L. Bers, Theory of pseudo-analytic functions, New York University, 1953. MR 15,211c Zbl 0051.31603

[Bers 1956] L. Bers, "An outline of the theory of pseudoanalytic functions", Bull. Amer. Math. Soc. 62 (1956), 291-331. MR 18,470d Zbl 0072.07703

[Bers 1977] L. Bers, "Quasiconformal mappings, with applications to differential equations, function theory and topology", Bull. Amer. Math. Soc. 83:6 (1977), 1083-1100. MR 57 \#3384 Zbl 0419.30016

[Bryant et al. 1991] R. L. Bryant, S. S. Chern, R. B. Gardner, H. L. Goldschmidt, and P. A. Griffiths, Exterior differential systems, Mathematical Sciences Research Institute Publications 18, Springer, New York, 1991. MR 92h:58007 Zbl 0726.58002

[Douglis and Nirenberg 1955] A. Douglis and L. Nirenberg, "Interior estimates for elliptic systems of partial differential equations", Comm. Pure Appl. Math. 8 (1955), 503-538. MR 17,743b Zbl 0066.08002

[Gardner 1967] R. B. Gardner, "Invariants of Pfaffian systems", Trans. Amer. Math. Soc. 126 (1967), 514-533. MR 35 \#2233 Zbl 0161.41301

[Gilbarg and Trudinger 1998] D. Gilbarg and N. S. Trudinger, Elliptic partial differential equations of second order, revised 2nd ed., Grundlehren der Math. Wissenschaften 224, Springer, Berlin, 1998. MR 2001k:35004 Zbl 1042.35002

[Goldschmidt 1967] H. Goldschmidt, "Existence theorems for analytic linear partial differential equations”, Ann. of Math. (2) 86 (1967), 246-270. MR 36 \#2933 Zbl 0154.35103

[Han and Kim 2012] C.-K. Han and H. Kim, "Holomorphic functions on almost complex manifolds", J. Korean Math. Soc. 49:2 (2012), 379-394. MR 2933604 Zbl 1238.32017

[Han and Lee 2010] C.-K. Han and K.-H. Lee, "Integrable submanifolds in almost complex manifolds”, J. Geom. Anal. 20:1 (2010), 177-192. MR 2011b:32044 Zbl 1185.32015

[Han and Park 2013] C.-K. Han and J.-D. Park, "Method of characteristics and first integrals for systems of quasi-linear partial differential equations of first order", 2013. Submitted for publication.

[Hartogs 1906] F. Hartogs, "Einige Folgerungen aus der Cauchyschen Integralformei bei Funktionen mehrerer Veränderlichen”, pp. 223-242 in Sitzungsberichte der Königlich Bayerischen Akademie der Wissenschaften (Münch), Mathematisch-Physikalische Klasse 36, München, Berlin, 1906. JFM 37.0443.01

[Hayashi 1996] Y. Hayashi, "A global theory of some nonlinear Cauchy-Riemann system in several complex variables”, Math. Nachr. 178 (1996), 157-198. MR 97d:32023 Zbl 0855.35091

[Hörmander 1965] L. Hörmander, " $L^{2}$ estimates and existence theorems for the $\bar{\partial}$ operator", Acta Math. 113 (1965), 89-152. MR 31 \#3691 Zbl 0158.11002

[Kohn 1963] J. J. Kohn, "Harmonic integrals on strongly pseudo-convex manifolds, I", Ann. of Math. (2) 78 (1963), 112-148. MR 27 \#2999 Zbl 0161.09302

[Kruglikov 1998] B. S. Kruglikov, "Nijenhuis tensors and obstructions to the construction of pseudoholomorphic mappings", Math. Notes 63:4 (1998), 476-493. MR 2000f:32036 Zbl 0933.32038

[MacKichan 1968] B. B. MacKichan, A generalization to overdetermined systems of the notion of diagonal operators, Ph.D. thesis, Stanford University, 1968. MR 2617842

[Monge 1803] G. Monge, "Mémoire sur la théorie d'une équation aux dérivées partielles du premier ordre", Journal de l'École Polytechnique 9 (1803), 56-99. 
[Morrey 1938] C. B. Morrey, Jr., "On the solutions of quasi-linear elliptic partial differential equations”, Trans. Amer. Math. Soc. 43:1 (1938), 126-166. MR 1501936 Zbl 0018.40501

[Mushkarov 1981] O. K. Mushkarov, "Almost complex manifolds without almost holomorphic functions", C. R. Acad. Bulgare Sci. 34:9 (1981), 1225-1228. MR 83i:53058 Zbl 0487.53030

[Mushkarov 1986] O. K. Mushkarov, "Existence of holomorphic functions on almost complex manifolds", Math. Z. 192:2 (1986), 283-295. MR 87i:53055 Zbl 0581.53025

[Newlander and Nirenberg 1957] A. Newlander and L. Nirenberg, "Complex analytic coordinates in almost complex manifolds", Ann. of Math. (2) 65 (1957), 391-404. MR 19,577a Zbl 0079.16102

[Nijenhuis and Woolf 1963] A. Nijenhuis and W. B. Woolf, "Some integration problems in almostcomplex and complex manifolds", Ann. of Math. (2) 77 (1963), 424-489. MR 26 \#6992 Zbl 0115: 16103

[Oka 1953] K. Oka, "Sur les fonctions analytiques de plusieurs variables, IX: Domaines finis sans point critique intérieur", Jap. J. Math. 23 (1953), 97-155. MR 17,82b Zbl 0053.24302

[Quillen 1964] D. G. Quillen, Formal properties of over-determined systems of linear partial differential equations, Ph.D. thesis, Harvard University, 1964. MR 2939514

[So'n 1990] L. H. So'n, “Extension problem in generalized complex analysis”, pp. 215-229 in Functional analytic methods in complex analysis and applications to partial differential equations (Trieste, 1988), edited by A. S. A. Mshimba and W. Tutschke, World Sci., River Edge, NJ, 1990. MR 93c:32019 Zbl 0947.30502

[Spencer 1962] D. C. Spencer, "Deformation of structures on manifolds defined by transitive, continuous pseudogroups, I: Infinitesimal deformations of structure", Ann. of Math. (2) 76 (1962), 306-398. MR 27 \#6287a Zbl 0124.38601

[Spencer 1965] D. C. Spencer, "Deformation of structures on manifolds defined by transitive, continuous pseudogroups, III: Structures defined by elliptic pseudogroups", Ann. of Math. (2) 81 (1965), 389-450. MR 31 \#4052 Zbl 0192.29603

[Spencer 1969] D. C. Spencer, "Overdetermined systems of linear partial differential equations", Bull. Amer. Math. Soc. 75 (1969), 179-239. MR 39 \#3533 Zbl 0185.33801

[Sweeney 1968] W. J. Sweeney, “The D-Neumann problem”, Acta Math. 120 (1968), 223-277. MR 37 \#2250 Zbl 0159.38402

[Taylor 1997] M. E. Taylor, Partial differential equations, III: Nonlinear equations, Applied Mathematical Sciences 117, Springer, New York, 1997. MR 98k:35001 Zbl 0869.35004

[Trèves 1981] F. Trèves, Approximation and representation of functions and distributions annihilated by a system of complex vector fields, École Polytechnique Centre de Mathématiques, Palaiseau, 1981. MR 84k:58008 Zbl 0515.58030

[Vekua 1962] I. N. Vekua, Generalized analytic functions, Pergamon, London, 1962. MR 27 \#321 Zbl 0100.07603

Received June 28, 2012. Revised September 26, 2012.

\section{CHONG-KYU HAN}

DEPARTMENT OF MATHEMATICS

SEOUL NATIONAL UNIVERSITY

SAN 56-1, SHILLYM-DONG, GWANAK-GU

SEOUL 151-742

SOUTH KOREA

ckhan@snu.ac.kr 
JONG-DO PARK

DEPARTMENT OF MATHEMATICS AND RESEARCH InSTITUTE FOR BASIC SCIENCES KYUNG HEE UNIVERSITY

SEOUL 130-701

SOUTH KOREA

mathjdpark@khu.ac.kr 


\title{
PACIFIC JOURNAL OF MATHEMATICS
}

\author{
msp.org/pjm
}

Founded in 1951 by E. F. Beckenbach (1906-1982) and F. Wolf (1904-1989)

\section{EDITORS}

V. S. Varadarajan (Managing Editor)

Department of Mathematics

University of California

Los Angeles, CA 90095-1555

pacific@math.ucla.edu

Paul Balmer

Department of Mathematics

University of California

Los Angeles, CA 90095-1555

balmer@math.ucla.edu

Daryl Cooper

Department of Mathematics

University of California

Santa Barbara, CA 93106-3080 cooper@math.ucsb.edu

Jiang-Hua $\mathrm{Lu}$

Department of Mathematics

Pokfulam Rd., Hong Kong jhlu@maths.hku.hk
The University of Hong Kong

Don Blasius

Department of Mathematics University of California

Los Angeles, CA 90095-1555

blasius@math.ucla.edu

Robert Finn

Department of Mathematics Stanford University

Stanford, CA 94305-2125

finn@math.stanford.edu

Sorin Popa

Department of Mathematics

University of California

Los Angeles, CA 90095-1555 popa@math.ucla.edu

Paul Yang

Department of Mathematics Princeton University

Princeton NJ 08544-1000

yang@math.princeton.edu

\section{PRODUCTION}

Silvio Levy, Scientific Editor, production@msp.org

\section{SUPPORTING INSTITUTIONS}

ACADEMIA SINICA, TAIPEI

CALIFORNIA INST. OF TECHNOLOGY

INST. DE MATEMÁTICA PURA E APLICADA

KEIO UNIVERSITY

MATH. SCIENCES RESEARCH INSTITUTE

NEW MEXICO STATE UNIV.

OREGON STATE UNIV.

\author{
STANFORD UNIVERSITY \\ UNIV. OF BRITISH COLUMBIA \\ UNIV. OF CALIFORNIA, BERKELEY \\ UNIV. OF CALIFORNIA, DAVIS \\ UNIV. OF CALIFORNIA, LOS ANGELES \\ UNIV. OF CALIFORNIA, RIVERSIDE \\ UNIV. OF CALIFORNIA, SAN DIEGO \\ UNIV. OF CALIF., SANTA BARBARA
}

\author{
Vyjayanthi Chari \\ Department of Mathematics \\ University of California \\ Riverside, CA 92521-0135 \\ chari@math.ucr.edu \\ Kefeng Liu \\ Department of Mathematics \\ University of California \\ Los Angeles, CA 90095-1555 \\ liu@math.ucla.edu \\ Jie Qing \\ Department of Mathematics \\ University of California \\ Santa Cruz, CA 95064 \\ qing@cats.ucsc.edu
}

These supporting institutions contribute to the cost of publication of this Journal, but they are not owners or publishers and have no responsibility for its contents or policies.

See inside back cover or msp.org/pjm for submission instructions.

The subscription price for 2013 is US \$400/year for the electronic version, and \$485/year for print and electronic.

Subscriptions, requests for back issues and changes of subscribers address should be sent to Pacific Journal of Mathematics, P.O. Box 4163, Berkeley, CA 94704-0163, U.S.A. The Pacific Journal of Mathematics is indexed by Mathematical Reviews, Zentralblatt MATH, PASCAL CNRS Index, Referativnyi Zhurnal, Current Mathematical Publications and the Science Citation Index.

The Pacific Journal of Mathematics (ISSN 0030-8730) at the University of California, c/o Department of Mathematics, 798 Evans Hall \#3840, Berkeley, CA 94720-3840, is published twelve times a year. Periodical rate postage paid at Berkeley, CA 94704, and additional mailing offices. POSTMASTER: send address changes to Pacific Journal of Mathematics, P.O. Box 4163, Berkeley, CA 94704-0163.

PJM peer review and production are managed by EditFLOW ${ }^{\circledR}$ from Mathematical Sciences Publishers.

\section{PUBLISHED BY}

mathematical sciences publishers

nonprofit scientific publishing

http://msp.org/

(C) 2013 Mathematical Sciences Publishers 


\section{PACIFIC JOURNAL OF MATHEMATICS}

Volume $265 \quad$ No. $1 \quad$ September 2013

Genus-two Goeritz groups of lens spaces

SANGBUM CHO

A compact embedding theorem for generalized Sobolev spaces

SENG-KeE ChUa, SCOTT Rodney and Richard L. WheEden

Partial integrability of almost complex structures and the existence of

solutions for quasilinear Cauchy-Riemann equations

CHONG-KYU HAN and JONG-DO PARK

An overdetermined problem in potential theory

DMITRY KHAVINSON, ERIK LUNDBERG and RAZVAN TEODORESCU

Quasisymmetric homeomorphisms on reducible Carnot groups

XIANGDONG XIE

Capillarity and Archimedes' principle

JOHN MCCUAN and RAY TREINEN

Generalized eigenvalue problems of nonhomogeneous elliptic operators and their application

DUmitru Motreanu and Mieko TANaKa

Weighted Ricci curvature estimates for Hilbert and Funk geometries

SHIN-ICHI OHTA

On generalized weighted Hilbert matrices

EMMANUEL PREISSMANN and OLIVIER LÉVÊQUE

Unique prime decomposition results for factors coming from wreath product 221 groups

J. OWEN SizEMORE and ADAM WinCHESTER

On volume growth of gradient steady Ricci solitons

Guofang Wei and Peng Wu

Classification of moduli spaces of arrangements of nine projective lines

FEI YE 